\title{
FAIR PRICING OF LIFE \\ INSURANCE PARTICIPATING POLICIES \\ WITH A MINIMUM INTEREST RATE GUARANTEED
}

BY

AnNA Rita BACINELLO

Dipartimento di Matematica Applicata alle Scienze Economiche

Statistiche ed Attuariali "Bruno de Finetti",

Università degli Studi di Trieste, Trieste, Italy.

\begin{abstract}
In this paper we analyse, in a contingent-claims framework, one of the most common life insurance policies sold in Italy during the last two decades. The policy, of the endowment type, is initially priced as a standard one, given a mortality table and a technical interest rate. Subsequently, at the end of each policy year, the insurance company grants a bonus, which is credited to the mathematical reserve and depends on the performance of a special investment portfolio. More precisely, this bonus is determined in such a way that the total interest rate credited to the insured equals a given percentage (participation level) of the annual return on the reference portfolio and anyway does not fall below the technical rate (minimum interest rate guaranteed, henceforth). Moreover, if the contract is paid by periodical premiums, it is usually stated that the annual premium is adjusted at the same rate of the bonus, and thus the benefit is also adjusted in the same measure. In such policy the variables controlled by the insurance company (control-variables, henceforth) are the technical rate, the participation level and, in some sense, the riskiness of the reference portfolio measured by its volatility. However, as it is intuitive, not all sets of values for these variables give rise to a fair contract, i.e. to a contract priced consistently with the usual assumptions on financial markets and, in particular, with no-arbitrage. We derive then necessary and sufficient conditions under which each control-variable is determined by a fair pricing of the contract, given the remaining two ones.
\end{abstract}

\section{KEYWORDS}

Policies with profits, Minimum guarantee, Fair pricing, Black and Scholes framework. 


\section{INTRODUCTION}

At the end of the seventies a new kind of life insurance product, the so-called rivalutabile, was introduced in Italy, together with the index-linked policies ${ }^{1}$, in order to match the high level of inflation that led the returns on Treasury Bonds and fixed-income securities up to $20 \%$ p.a.. The interest rate of 3\% p.a. commonly guaranteed by traditional life insurance products was indeed completely inadequate and seriously jeopardized the marketability of such products.

The term rivalutabile identifies the Italian version of the widely known participating policy, or policy with profits (Universal Life Insurance, in the United States). In Italy a special portfolio of investments, covering at least the mathematical reserves of all the policies with profits issued by a same insurance company, is constituted and kept apart from the other assets of the company. Within the end of each calendar year the rate of return on this portfolio (reference portfolio, henceforth) in the preceding financial year is computed and certified by a special auditor. The financial year usually begins on November 1st and ends on October 31st. A percentage of this rate of return, that is defined every year and usually cannot be less than a fixed level (e.g. 70\%), is granted to the insured. More precisely, if the granted rate of return exceeds the technical interest rate already included in the premium calculation, a bonus computed at the excess rate is credited to the mathematical reserves of all the participating policies when they reach their anniversary (i.e., at the end of the policy year). Observe that, in this way, the technical rate becomes a minimum interest rate guaranteed.

Policies with profits are very often paid by annual premiums. If this is the case, it is usually stated that the annual premium increases at the same excess rate credited to the mathematical reserve so that, as like as in the single premium contracts, also the benefits are adjusted in the same measure in order to maintain the actuarial equilibrium with regard to the residual policy period.

Since the pioneering work by Brennan and Schwartz $(1976,1979 a, 1979 b)$ and Boyle and Schwartz (1977), a great prominence has been given so far in the financial and actuarial literature to the issues of pricing and hedging equity-linked life insurance contracts with minimum guarantees. In contrast with this, participating policies have not been studied very much in a contingent-claims framework ${ }^{2}$, although they are the most important life insurance products in terms of market size. This is probably due to the fact that the minimum interest rate guaranteed used to be far lower than the market rates, and therefore the risk associated to the issue of the guarantee seemed to be quite negligible and was not seriously considered a threat to the solvency of a life insurance company. Now that the economic setting has dramatically capsized in most industrial countries and the market interest rates have sunk

\footnotetext{
Actually, the first index-linked policy traded in Italy dates back to 1968 .

2 The first application of option pricing theory to bonuses on participating policies of which we are aware is Wilkie (1987).
} 
up to very low levels ${ }^{3}$, this threat has become impending. Then an accurate assessment of all the parameters characterizing the guarantees and the bonus mechanism constitutes a crucial problem in the management of a life insurance company.

Some recent contributions in this direction are due to Briys and de Varenne (1997), Miltersen and Persson (2000a, 2000b), Grosen and Jørgensen (2000), Hansen and Miltersen (2000), Jensen, Jørgensen and Grosen (2000).

Briys and de Varenne (1997) consider a single-period valuation model for the equities and the liabilities of a life insurance company. In particular the policyholders, i.e., the "owners" of the liabilities, earn a minimum interest rate guaranteed plus a bonus. The bonus is given by a percentage (participation level) of the difference, if positive, between the final value of the assets times the initial ratio between liabilities and assets, and the minimum guaranteed final value of liabilities. In their valuation model Briys and de Varenne take into account also the risk of default. Under the assumption that the assets follow a lognormal process and the stochastic interest rates behave as in Vasicek (1977), they obtain a closed-form solution both for equities and for liabilities. They also derive an equilibrium condition which relates, by an explicit formula, the participation level to the minimum interest rate guaranteed.

Miltersen and Persson (2000a) consider a multiperiod valuation model in which the "customers" (i.e., the policyholders) are entitled to two different accounts: the "customer's account" and the "bonus account". The customer's account earns, at the end of each year, a minimum interest rate guaranteed plus a percentage of the positive excess between the realized rate of return on a benchmark portfolio and the promised minimum rate. The bonus account, instead, is a sort of buffer that receives, in "good" years, an additional percentage of the positive difference between the above mentioned rates and, in "bad" years, is used for fulfilling the minimum guarantee promise. At maturity, if the bonus account is negative, the deficit is anyway absorbed by the insurance company. Under the Black and Scholes (1973) framework, Miltersen and Persson derive a closed-form solution for the customer's account and use instead the Monte Carlo approach for valuing the bonus account. They also derive an equilibrium condition which relates the participation levels, the volatility parameter characterizing the return on the benchmark, and the annual minimum interest rates guaranteed.

Grosen and Jørgensen (2000) consider, as Miltersen and Persson, a multiperiod valuation model, and split the Liability Side of the Balance Sheet into two components: the "policy reserve" and the "bonus reserve" (or simply "buffer"). At the end of each policy year the policy reserve earns the maximum between a minimum interest rate guaranteed and a percentage of the (positive) difference between the ratio buffer/policy reserve valued at the end of the preceding year and a target buffer ratio. Also Grosen and Jørgensen

\footnotetext{
3 E.g., the return on 1-year Italian Treasury zero-coupon-bonds was about 2.3\% p.a. from mid-April to mid-May 1999
} 
model the assets à la Black and Scholes. In particular, they show how a typical participating contract can be decomposed into a risk-free bond element, a bonus option (of European style), and a surrender option (of American style). Then they separately price these elements by using a Monte Carlo approach for the European option and a binomial lattice approach for the American one.

The remaining papers constitute further extensions of the results by Grosen and Jorgensen (2000) and Miltersen and Persson (2000a).

All the above mentioned authors consider a single-payment contract in which the mortality risk is not taken into account. The purpose of this paper is the fair pricing of an actual life insurance participating policy that couples the mortality risk with the financial elements and is paid either by a single premium or by a sequence of periodical premiums.

The policy, of the endowment type, exhibits almost all the features of the Italian products, and in particular the same pricing technique. This technique consists in computing the (initial) net premium, single or annual, as in the case of a standard endowment policy, given the initial sum insured (benefit), the technical interest rate, and a mortality table from which the life and death probabilities are extracted; hence the financial risk connected to the technical rate guarantee is completely disregarded. Then, at the end of each policy year, the benefit and the periodical premium are adjusted according to the bonus mechanism.

By "fair pricing" we mean pricing consistent with no-arbitrage in the financial markets. Therefore, since the rules for computing the premium(s) are anyway fixed, a fair pricing is feasible by suitably choosing the parameters characterizing the contract. The contractual parameters, "controlled" by the insurance company, are the participation level and the technical (or minimum guaranteed) interest rate. Another parameter which, in some sense, can be also "controlled" by the insurance company is the riskiness of the investments composing the reference portfolio, measured by a volatility coefficient. If, in particular, this volatility is high, the reference portfolio can produce high returns as like as heavy losses. The losses, however, are entirely suffered by the insurance company since the policyholder benefits of the minimum interest rate guarantee. Then, in this case, the chance of high bonus returns may induce the policyholder to accept a lower minimum rate guaranteed and/or a lower participation level. Moreover, it is quite intuitive that there is also a trade-off between the participation level and the minimum rate.

We suggest that the insurance company, instead of keeping together the investments concerning all the participating policies issued, graduates several reference portfolios according to their volatility, and thus offers its customers the choice among different triplets of technical rate, participation level, volatility.

Under the Black and Scholes assumption for the evolution of the reference portfolio and assuming independence between mortality risk and financial risk, we express, first of all, the fair price of a participating contract in terms of one-year call options. This has some similarities with tandem options (see, e.g., Blazenko, Boyle and Newport (1990)) and the ratchet features of some 
equity-linked life insurance policies. Then we derive a very simple closed-form fairness relation, the same both in the case of a single premium and in that of periodical premiums. We also give necessary and sufficient conditions under which each one of the three control-parameters is determined, given the remaining two ones and the market instantaneous riskless interest rate. These solutions turn out to be unique and quasi-explicit.

The paper is organized as follows. In Section 2 we formalize the structure of the policy and of the bonus mechanism. Section 3 starts with the presentation of our valuation framework and ends with the definition of the arbitrage condition. In Section 4 we derive the fairness relation and give the conditions under which each control-parameter is uniquely determined; moreover, we present some numerical examples of sets of parameters satisfying this relation. In Section 5 we hint at possible hedging strategies and at some practical problems that they could involve. Section 6 concludes the paper.

\section{THE STRUCTURE OF THE POLICY}

Consider a single endowment policy (or a cohort of identical endowment policies) issued at time 0 and maturing at time $T$. We denote by $\mathrm{x}$ the entry age, by $\mathrm{C}_{0}$ the "initial" sum insured, and by $\mathrm{i}$ the annual compounded technical interest rate. In what follows we disregard any problem connected with expenses and relative loadings, so that only net premiums are involved.

\subsection{Single premium contracts}

If the policy is paid by a single amount $\mathrm{U}$ at the initiation of the contract, and the benefit is assumed to be due at the end of the year of death $t=1,2, \ldots, T$ or, at the latest, at maturity $T$, the following relation defines $U$ :

$$
\mathrm{U}=\mathrm{C}_{0} \mathrm{~A}_{\mathrm{x}: \mathrm{i}} \frac{(\mathrm{i})}{\mathrm{T} \mid}=\mathrm{C}_{0}\left(\sum_{\mathrm{t}=1}^{\mathrm{T}-1} \mathrm{t}-1 \mid \mathrm{q}_{\mathrm{x}} \mathrm{v}^{\mathrm{t}}+{ }_{\mathrm{T}-1} \mathrm{p}_{\mathrm{x}} \mathrm{v}^{\mathrm{T}}\right)
$$

where $v=(1+i)^{-1}, t_{-1} \mid{ }_{1} q_{x}$ represents the probability that the insured dies during the $t-t h$ year of contract (i.e., between times $t-1$ and $t$ ), and ${ }_{T-1} p_{x}$ represents the probability that the insured is alive at time $\mathrm{T}-1$ (i.e., he(she) dies during the last year of contract or survives the term of the contract).

Observe that the premium $U$ is expressed as an expected value of the discounted benefit, just as though the insurance company were risk-neutral with respect to mortality. Since mortality fluctuations, due to (a) uncertain future mortality improvements and (b) parameter uncertainty for a given company, actually occur, the insurer (which is not risk-neutral but risk-averse) usually requests a compensation for accepting mortality risk. Traditionally this compensation is not explicitly added to the premium, but it is implied by the 
choice of a "safe" mortality table according to which the premium, computed as an expected value, is implicitly charged by a "safety loading". Then the life and death probabilities extracted from this table usually differ from the "true" ones, unless the insurance company is able to eliminate (by an extreme diversification) mortality fluctuations and operates in a perfectly competitive market. Hence this "adjusted" table may be interpreted as a "risk-neutral" one, in the sense that the term "risk-neutrality" has in the Financial Economics environment.

We assume that, at the end of the t-th policy year, if the contract is still in force, the mathematical reserve is adjusted at a rate $\delta_{t}$ ("bonus rate") defined as follows:

$$
\delta_{\mathrm{t}}=\max \left\{\frac{\eta g_{\mathrm{t}}-\mathrm{i}}{1+\mathrm{i}}, 0\right\}, \quad \mathrm{t}=1,2, \ldots, \mathrm{T}
$$

The parameter $\eta$, between 0 and 1 , denotes the participation level, for simplicity assumed to be constant in time, and $\mathrm{g}_{\mathrm{t}}$ denotes the annual return on the reference portfolio. Relation (2) formally translates the fact that the total interest rate credited to the mathematical reserve during the t-th policy year, $(1+i)\left(1+\delta_{t}\right)-1$, equals the maximum between $i$ and $\eta g_{t}$, i.e., that $i$ is a minimum rate of return guaranteed to the policyholder.

Since we are dealing with a single premium contract, the bonus credited to the mathematical reserve implies a proportional adjustment, at the rate $\delta_{t}$, also of the sum insured. In particular, if the insured dies within the term of the contract, we assume that the benefit profits by an additional (last) adjustment just before being paid at the end of the year of death. This is in contrast with what happens in Italy for participating policies, where the amount of the benefit due in a given policy year is fixed at the beginning of the year and therefore there is a sort of predictability with respect to the relevant information characterizing the financial uncertainty. We point out that our assumption is not motivated by the wish of obtaining closed-form solutions since, under the valuation framework depicted in the next section, the market value of the policy would anyway be expressible in closed-form. However, as we will see in the sequel of the paper, it is just this assumption that allows us to derive a very simple and explicit fairness relation, depending only on four variables.

Denoting by $C_{t}, t=1,2, \ldots, T$, the benefit paid at time $t$ if the insured dies between ages $x+t-1$ and $x+t$ or, for $t=T$, in case of survival, the following recursive relation links then the benefits of successive years:

$$
\mathrm{C}_{\mathrm{t}}=\mathrm{C}_{\mathrm{t}-1}\left(1+\delta_{\mathrm{t}}\right), \quad \mathrm{t}=1,2, \ldots, \mathrm{T} .
$$

The iterative expression for them is instead:

$$
\mathrm{C}_{\mathrm{t}}=\mathrm{C}_{0} \prod_{\mathrm{j}=1}^{\mathrm{t}}\left(1+\delta_{\mathrm{j}}\right), \quad \mathrm{t}=1,2, \ldots, \mathrm{T} .
$$




\subsection{Periodic premium contracts}

Assume now that the policy is paid by a sequence of periodical premiums, due at the beginning of each year of contract, if the insured is alive. The initial premium, $\mathrm{P}_{0}$, paid at time 0 , is given by

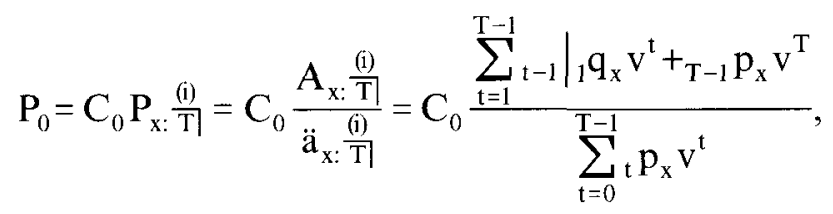

where the death probabilities ${ }_{t-1} \mid{ }_{l} q_{x}$ and the survival probabilities ${ }_{t} p_{x}$ are extracted from the same table introduced in the previous subsection. Moreover, most of the considerations and assumptions made in that subsection are still valid, in particular the bonus mechanism described by relation (2).

In Italy it is usual that the periodical premium of a participating policy is annually adjusted at the same bonus rate $\delta_{\mathrm{t}}$ credited to the mathematical reserve. In this case, denoting by $P_{t}, t=1,2, \ldots, T-1$, the $(t+1)$-th premium paid at time $t$, if the insured is alive, one has

$$
\mathrm{P}_{\mathrm{t}}=\mathrm{P}_{\mathrm{t}-1}\left(1+\delta_{\mathrm{t}}\right), \quad \mathrm{t}=1,2, \ldots, \mathrm{T}-1 .
$$

or, alternatively,

$$
P_{t}= \begin{cases}P_{0} & t=0 \\ P_{0} \prod_{j=1}^{t}\left(1+\delta_{j}\right) & t=1,2, \ldots, T-1\end{cases}
$$

If this is the case, the benefit $C_{t}$ is also adjusted in the same measure, so that relation (3) or, alternatively, (4), still holds.

In this paper we also make the assumption of identical adjustment rates for the mathematical reserve and the premium (and hence for the benefit). However, we observe that not all countries (for example, the UK) update premiums as happens in this model. Moreover, also for the Italian contracts it is sometimes stated that the adjustment rate of the periodical premium is only a fraction, for instance one half, of $\delta_{t}$, or even 0 (i.e., the premiums are constant). In these cases an actuarial equilibrium relation concerning the residual policy period imposes that the adjustment rate of the benefit in a given year is a weighted mean of the remaining two adjustment rates in the same year (see, e.g., Pentikäinen (1968)). Unfortunately this mean turns out to be path-dependent since it depends (through the updated values of the mathematical reserve and the premium) on all the adjustment rates in the past years. Therefore it is hard to obtain closed-form relations for the market value of the contract in these cases, hence our assumption of identical rates is crucial in the derivation of all the results concerning periodic premium contracts presented in the next sections. 


\section{The VAluation MODEL}

In this section we describe, first of all, the basic assumptions concerning the financial set-up. Then, observing that both the periodical premiums and the benefit are typical contingent-claims, we apply the martingale approach put forward by Harrison and Kreps (1979) and Harrison and Pliska (1981, 1983) to obtain a valuation formula for them. Finally, the mortality risk comes into play in order to establish a fairness condition in the pricing of the contract.

\subsection{Assumptions}

We assume perfectly competitive, frictionless and arbitrage-free securities markets, populated by rational and non-satiated agents, all sharing the same information. We denote by $\mathrm{r}$ the continuously compounded market rate, assumed to be deterministic and constant. Therefore, in our framework, there is a unique source of financial uncertainty, reflected by a stochastic evolution of the reference portfolio. Assume that this uncertainty is generated by a standard brownian motion $\mathrm{W}$, defined on a filtered probability space $(\Omega, \mathfrak{s}, \mathrm{Q})$ in the time interval $[0, T]$. In particular, $Q$ represents the equivalent martingale measure, under which the discounted price of any financial security is a martingale (see Harrison and Kreps (1979)).

We assume that the reference portfolio is a well-diversified one, and is split into shares, or units. Moreover, dividends, coupons or whatever else yielded by the assets composing it are immediately reinvested in the same portfolio and thus contribute to increase its unit price. Therefore its annual returns are completely determined by the evolution of its unit price and not by that of its total value, which reflects also new investments (corresponding, for instance, to the payment of periodical premiums or to the entry of new policies into the portfolio) and withdrawals (when some policy expires). We denote by $G_{t}$ the unit price at time $t$ of the reference portfolio and model it, under $Q$, as a geometric brownian motion:

$$
\frac{\mathrm{dG}_{\mathrm{t}}}{\mathrm{G}_{\mathrm{t}}}=\mathrm{rdt}+\sigma \mathrm{dW}_{\mathrm{t}}, \quad \mathrm{t} \in[0, \mathrm{~T}],
$$

with the constant $\sigma$ representing the volatility parameter and $\mathrm{G}_{0}$ given. As it is well known, the solution to the stochastic differential equation (8) is given by

$$
\mathrm{G}_{\mathrm{t}}=\mathrm{G}_{0} \exp \left\{\left(\mathrm{r}-\sigma^{2} / 2\right) \mathrm{t}+\sigma \mathrm{W}_{\mathrm{t}}\right\}, \quad \mathrm{t} \in[0, \mathrm{~T}] .
$$

We assume that the annual compounded rates of return $g_{t}$ introduced in the previous section are defined as

$$
\mathrm{g}_{\mathrm{t}}=\frac{\mathrm{G}_{\mathrm{t}}}{\mathrm{G}_{\mathrm{t}-1}}-1, \quad \mathrm{t}=1,2, \ldots, \mathrm{T}^{4},
$$

\footnotetext{
4 As described in the Introduction, the annual rate of return on the reference portfolio for Italian participating policies is actually referred to a financial year, that generally ends at least two months before a policy year. Here, for simplicity, we have instead assumed that $g_{t}$ is referred to a policy year.
} 
so that $1+\mathrm{g}_{\mathrm{t}}=\exp \left\{\mathrm{r}-\sigma^{2} / 2+\sigma\left(\mathrm{W}_{\mathrm{t}}-\mathrm{W}_{\mathrm{t}-1}\right)\right\}$ are independent and identically distributed (i.i.d.) for $\mathrm{t}=1,2, \ldots, \mathrm{T}$ and their logarithms, representing continuously compounded rates of return, are all independent and normally distributed with mean $r-\sigma^{2} / 2$ and variance $\sigma^{2}$. Therefore also the bonus rates $\delta_{t}$ defined by relation (2) of Section 2 turn out to be i.i.d. As we will see in a moment, this fact is really crucial since the independence of the bonus rates allows us to express the market value of the contract in terms of one-year call options and, together with the identical distribution, to translate the fairness condition into a very simple equation.

Finally, we assume independence between mortality and the financial elements, so that the valuation of the contract can be performed in two separate stages: in the first stage premiums and benefits defined by relations (7) and (4) of Section 2 are priced as like as they were (purely-financial) contingentclaims due with certainty at a fixed (future) date; in the second stage their time 0 prices are "weighted" with the probabilities introduced in Section 2 in order to get a "fair" price of the contract.

\subsection{Fair valuation of single premium contracts}

To value these contracts, we first need to compute, for any $t=1,2, \ldots, T$, the market value of the contingent-claim $C_{t}$, defined by relation (4) of the previous section and assumed to be due with certainty at time t. To this end we exploit the martingale approach put forward by Harrison and Kreps (1979) and Harrison and Pliska $(1981,1983)$ and express the time 0 price of $\mathrm{C}_{t}$, denoted by $\pi\left(\mathrm{C}_{t}\right)$, as the following expectation under the risk-neutral measure Q:

$$
\pi\left(C_{t}\right)=E^{Q}\left[\exp \{-r t\} C_{t}\right], \quad t=1,2, \ldots T .
$$

Exploiting relations (4) and (2) of Section 2 together with the stochastic independence of the bonus rates $\delta_{j}$ for $j=1,2, \ldots, T$, and after some algebraic manipulations, we get then

$\pi\left(C_{t}\right)=C_{0} \prod_{j=1}^{t}\left(\exp \{-r\}+\frac{\eta}{1+i} E^{Q}\left[\exp \{-r\} \max \left\{\left(1+g_{j}\right)-(1+i / \eta), 0\right\}\right]\right)$,

$\mathrm{t}=1, \ldots, \mathrm{T}$.

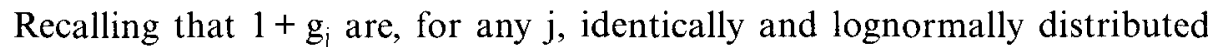
with, in particular, the same distribution as the time 1 stock price in the classical Black and Scholes (1973) model (given a time 0 price of the stock equal to 1 ), it is immediate to realize that the Q-expectation into the round brackets in the RHS of relation (12) represents the time 0 value of a European call option on a non dividend paying stock with initial price equal to 1 , option with maturity 1 and strike price equal to $1+\mathrm{i} / \eta$. Denoting this value by $\mathrm{c}$, we have then 


$$
\pi\left(C_{t}\right)=C_{0}\left(\exp \{-r\}+\frac{\eta}{1+i} c\right)^{t}, \quad t=1,2, \ldots T,
$$

with c given by the classical Black and Scholes (1973) formula:

$$
\mathrm{c}=\mathrm{F}\left(\mathrm{d}_{1}\right)-(1+\mathrm{i} / \eta) \exp \{-\mathrm{r}\} \mathrm{F}\left(\mathrm{d}_{2}\right),
$$

where $d_{1}=\frac{r+\sigma^{2} / 2-\ln (1+i / \eta)}{\sigma}, d_{2}=d_{1}-\sigma$, and $F$ denotes the cumulative distribution function of a standard normal variate.

The fair price of the single premium contract analysed in this paper, FVB, can be obtained by summing up, for $t=1,2, \ldots, T$, the time 0 values of $C_{1}$ weighted with the probabilities (introduced in Section 2 ) that they are exactly due at time t:

$$
F V B=C_{0}\left(\sum_{t=1}^{T-1}{ }_{t-1 \mid 1} q_{x} v_{*}^{t}+{ }_{T-1} p_{x} v_{*}^{T}\right)=C_{0} A_{x:} \frac{(i t)}{T},
$$

where $\mathrm{v}_{*}=\exp \{-\mathrm{r}\}+\frac{\eta}{1+\mathrm{i}} \mathrm{c}$ and $\mathrm{i}_{*}=\mathrm{v}_{*}^{-1}-1$.

Then the contract is fair if and only if the single premium $U$ equals FVB, i.e., recalling relation (i) of Section 2, if and only if the following condition is satisfied:

$$
A_{x:}: \frac{(i)}{T}=A_{x:}: \frac{(i *)}{T} \text {. }
$$

\subsection{Fair valuation of periodic premium contracts}

Most of what said in the previous subsection for single premium contracts is still valid in the case of periodical premiums. In particular the fair value of the benefit is still given by relation (15), while the fair value of the sequence of periodical premiums, FVP, is given by

$$
\mathrm{FVP}=\sum_{\mathrm{t}=0}^{\mathrm{T}-1} \mathrm{p}_{\mathrm{x}} \pi\left(\mathrm{P}_{\mathrm{t}}\right)
$$

where $\pi\left(P_{t}\right)=E^{Q}\left[\exp \{-r\} P_{t}\right]$ represents the time 0 price of the contingentclaim $P_{t}$, defined by relation (7) of Section 2 and assumed to be paid with certainty at time t. Exploiting the same arguments employed in the previous subsection, we have then

$$
\pi\left(P_{t}\right)=\left\{\begin{array}{ll}
P_{0} & t=0 \\
P_{0} v_{*}^{t} & t=1,2, \ldots T-1
\end{array},\right.
$$


so that

$$
\mathrm{FVP}=\mathrm{P}_{0} \sum_{\mathrm{t}=0}^{\mathrm{T}-1} \mathrm{t}_{\mathrm{x}} \mathrm{v}_{*}^{\mathrm{t}}=\mathrm{P}_{0} \ddot{\mathrm{a}}_{\mathrm{x}: \frac{(\mathrm{i})}{\mathrm{T}} \mid} \cdot
$$

The fairness requirement implies now that the fair value of the benefit, FVB, equals the fair value of the premiums, FVP, i.e., that

$$
\mathrm{C}_{0} \mathrm{~A}_{\mathrm{x}}: \frac{(\mathrm{i} *)}{\mathrm{T} T}=\mathrm{P}_{0} \ddot{\mathrm{a}}_{\mathrm{x}}: \frac{(\mathrm{i} *)}{\mathrm{T}} \mid \text {. }
$$

Recalling the definition of $\mathrm{P}_{0}$ given in relation (5) of Section 2, we conclude this subsection by stating that the contract is fair if and only if the following condition holds:

$$
P_{x: T}: \frac{(i)}{T}=P_{x: T}^{\left(i_{*}\right)},
$$

being $P_{x}: \frac{(i+x)}{T}=\frac{A_{x}: \frac{(i * x)}{T}}{\ddot{a}_{x}: \frac{\left(i_{*}\right)}{T} \mid}$,

\section{THE FAIRNESS RELATION}

We are now ready to characterize fair contracts by a very simple relation.

Proposition 1. A participating policy is fairly priced if and only if

$$
\exp \{-\mathbf{r}\}(1+\mathbf{i})+\eta \mathbf{c}-1=0 .
$$

Proof. In the previous section we have seen that a participating policy is fairly priced if and only if $\mathrm{K}(\mathrm{i})=\mathrm{K}\left(\mathrm{i}_{*}\right)$, being

$$
K(y)=A_{x:}: \frac{(y)}{T}\left|=\sum_{t=1}^{T-1}{ }_{t-1}\right| q_{x}(1+y)^{-t}{ }_{T-1} p_{x}(1+y)^{-T}
$$

for single premium contracts, and

$$
K(y)=P_{x:}: \frac{(y)}{T}=\frac{\sum_{t=1}^{T-1}{ }_{t-1} \mid{ }_{1} q_{x}(1+y)^{-t}+{ }_{T-1} p_{x}(1+y)^{-T}}{\sum_{t=0}^{T-1} p_{x}(1+y)^{-t}}
$$

for periodic premium ones (see relations (16) and (21) respectively). Since, in both cases, $\mathrm{K}$ is a strictly decreasing function of $\mathrm{y}$, then conditions (16) and (21) are both satisfied if and only if $i=i_{*}$, that is equivalent to relation (22). 
Note that relation (22) depends only on four parameters: the market instantaneous interest rate $r$, the annual compounded technical rate $i$, the participation level $\eta$, and the volatility coefficient $\sigma$. While the rate $r$ is exogenously given, the remaining parameters can be chosen by the insurance company, hence they are control-variables. In particular, $i$ and $\eta$ are directly fixed by the insurer, whereas $\sigma$ can be indirectly determined by a suitable choice of the assets that compose the reference portfolio.

It is quite intuitive that relation (22) defines a trade-off between any pair of control-parameters, given the third one and $r$. If the minimum interest rate guaranteed $i$ is high, then the insurance company cannot afford to fix a great participation level since, in "good" years (i.e., when $\mathrm{g}_{t}>\mathrm{i}$ ), it has to put aside a sufficient amount of non-distributed funds in order to be able to fulfil the minimum guarantee promise in "bad" years (when $\mathrm{g}_{t}<\mathrm{i}$ ). Similarly, a highly volatile reference portfolio can produce high returns as like as heavy losses. The losses, however, are entirely suffered by the insurer since the policyholder benefits of the minimum interest rate guarantee. Therefore in this case, to protect itself, the insurance company must keep the technical interest rate and/or the participation level down. In what follows this trade-off will formally turn out from the fact that all the partial derivatives with respect to the control-parameters i, $\eta, \sigma$ of the function

$$
g(r, i, \eta, \sigma):=\exp \{-r\}(1+i)+\eta c(r, i, \eta, \sigma)-1,
$$

with $\mathrm{c}(\mathrm{r}, \mathrm{i}, \eta, \sigma):=\mathrm{c}$ defined by relation (14), are of the same sign (in particular, positive).

In the remaining part of this section we will analyse, separately for each one of the three control-parameters, necessary and sufficient conditions under which there exists a unique solution to equation (22) for any given positive value of $r$ and once the insurance company has "fixed" the values of the other two control-parameters. Before doing this, however, we state the following

Proposition 2. A necessary condition for a fair pricing of the contract is

$$
\mathrm{i}<\exp \{\mathrm{r}\}-1
$$

or, equivalently,

$$
\ln (1+\mathrm{i})<\mathrm{r} .
$$

Proof. Observe that relation (22) is equivalent to

$$
c=\frac{1-\exp \{-r\}(1+i)}{\eta}
$$

Then Proposition 2 follows from the fact that the Black-Scholes value $\mathrm{c}$ is always strictly positive.

Q.E.D. 
Proposition 2 states that the technical interest rate $\mathrm{i}$ must be strictly less than the annual compounded market rate $\exp \{\mathbf{r}\}-1$ or, equivalently, that the continuously compounded technical rate, $\ln (1+i)$, must be less than $r$. Reminding that $\mathrm{i}$ is also the minimum interest rate guaranteed, this condition is indeed a quite obvious consequence of the no-arbitrage assumption.

\subsection{Solutions with respect to the technical rate i}

Given a market rate $r>0$, imagine that the insurance company has already fixed the participation level $\eta$, between 0 and 1 , and chosen the assets composing the reference portfolio, so that also $\sigma>0$ is given. We are now going to analyse if there exists a technical interest rate $i$, non negative and less than the annual compounded market rate $\exp \{r\}-1$, such that the fairness relation (22) holds, or, equivalently, such that the function $g$ defined by relation (23) equals 0 .

To this end observe, first of all, that

$$
\frac{\partial g}{\partial i}=\exp \{-r\}\left[1-F\left(d_{2}\right)\right]>0,
$$

i.e., that $\mathrm{g}$ is strictly increasing with respect to i. Moreover, since

$$
\sup _{i<\exp \{r\}-1} g(r, i, \eta, \sigma)=\lim _{i \rightarrow \exp \{r\}-1} g(r, i, \eta, \sigma)=\eta c(r, \exp \{r\}-1, \eta, \sigma)>0,
$$

then a necessary and sufficient condition under which there exists a unique solution to the equation $\mathrm{g}(\mathrm{r}, \mathrm{i}, \eta, \sigma)=0$, is

$$
\min _{i \geq 0} g(r, i, \eta, \sigma)=g(r, 0, \eta, \sigma)=\exp \{-r\}+\eta c(r, 0, \eta, \sigma)-1 \leq 0 .
$$

Substituting relation (14) of Section 3 for the Black-Scholes price, condition (28) becomes

$$
\eta \leq \frac{1-\exp \{-r\}}{F(r / \sigma+\sigma / 2)-\exp \{-r\} F(r / \sigma-\sigma / 2)}
$$

Observe that relation (29) defines an actual upper bound for $\eta$, i.e., that

$$
h(r, \sigma):=\frac{1-\exp \{-r\}}{F(r / \sigma+\sigma / 2)-\exp \{-r\} F(r / \sigma-\sigma / 2)}<1 .
$$

This is due to the facts that

$$
\frac{\partial \mathrm{h}}{\partial \sigma}=\frac{[\exp \{-\mathrm{r}\}-1] \exp \left\{-\frac{1}{2}\left(\mathrm{r}^{2} / \sigma^{2}+\sigma^{2} / 4+\mathrm{r}\right)\right\}}{\sqrt{2 \pi[\mathrm{F}(\mathrm{r} / \sigma+\sigma / 2)-\exp \{-\mathrm{r}\} \mathrm{F}(\mathrm{r} / \sigma-\sigma / 2)]^{2}}}<0,
$$


i.e., $\mathrm{h}$ is strictly decreasing with respect to $\sigma$, and

$$
\sup _{\sigma>0} h(r, \sigma)=\lim _{\sigma \rightarrow 0} h(r, \sigma)=1 .
$$

Summing up, given $r>0, \sigma>0$, and $\eta \in(0, h(r, \sigma)]$, there exists a unique $i \in$ $[0, \exp \{\mathrm{r}\}-1)$ such that the fairness relation holds.

To get a numerical insight, in Tables 1 and 2 we provide some examples of solutions to equation (22) with respect to i for given values of $\eta$ and $\sigma$. More precisely, the results reported in Table 1 are obtained by choosing a value of $3 \%$ for $r$, while Table 2 reports the results obtained when $r$ is equal to $10 \%$. We refer to Bacinello (2000) for similar results corresponding to different values of $r$ and to a wider range for $\sigma$.

TABLE 1

SOLUTIONS WITH RESPECT TO THE TECHNICAL RATE $\mathrm{j}$ (BASIS POINTS) WHHN $r=0.03$

\begin{tabular}{cccccccc}
\hline $\begin{array}{l}\boldsymbol{\eta} \\
\boldsymbol{\sigma}\end{array}$ & $\mathbf{0 . 1}$ & $\mathbf{0 . 2}$ & $\mathbf{0 . 3}$ & $\mathbf{0 . 4}$ & $\mathbf{0 . 5}$ & $\mathbf{0 . 6}$ & $\mathbf{0 . 7}$ \\
\hline $\mathbf{0 . 0 5}$ & 305 & 304 & 297 & 276 & 238 & 180 & 95 \\
$\mathbf{0 . 1 0}$ & 304 & 288 & 239 & 156 & 41 & & \\
$\mathbf{0 . 1 5}$ & 300 & 248 & 143 & & & \\
$\mathbf{0 . 2 0}$ & 289 & 193 & 28 & & & \\
$\mathbf{0 . 2 5}$ & 273 & 128 & & & & \\
$\mathbf{0 . 3 0}$ & 252 & 57 & & & & \\
$\mathbf{0 . 3 5}$ & 227 & & & & \\
$\mathbf{0 . 4 0}$ & 200 & &
\end{tabular}

TABLE 2

SOLUTIONS WITH RESPECT TO THE TECHNICAL RATE $i$ (BASIS POINTS) WHEN $r=0.1$

\begin{tabular}{ccrrrrrrrr}
\hline \hline $\begin{array}{l}\boldsymbol{\eta} \\
\boldsymbol{\sigma}\end{array}$ & $\mathbf{0 . 1}$ & $\mathbf{0 . 2}$ & $\mathbf{0 . 3}$ & $\mathbf{0 . 4}$ & $\mathbf{0 . 5}$ & $\mathbf{0 . 6}$ & $\mathbf{0 . 7}$ & $\mathbf{0 . 8}$ & $\mathbf{0 . 9}$ \\
\hline $\mathbf{0 . 0 5}$ & 1052 & 1052 & 1052 & 1051 & 1048 & 1031 & 990 & 905 & 726 \\
$\mathbf{0 . 1 0}$ & 1052 & 1052 & 1049 & 1030 & 982 & 893 & 750 & 524 & 138 \\
$\mathbf{0 . 1 5}$ & 1052 & 1049 & 1027 & 963 & 849 & 676 & 428 & 70 & \\
$\mathbf{0 . 2 0}$ & 1052 & 1039 & 981 & 862 & 677 & 419 & 71 & & \\
$\mathbf{0 . 2 5}$ & 1051 & 1019 & 916 & 737 & 482 & 143 & & & \\
$\mathbf{0 . 3 0}$ & 1048 & 989 & 837 & 598 & 274 & & & & \\
$\mathbf{0 . 3 5}$ & 1043 & 950 & 748 & 450 & 61 & & & & \\
$\mathbf{0 . 4 0}$ & 1035 & 904 & 652 & 297 & & & &
\end{tabular}

The results reported in Tables 1 and 2 do not require many comments. We only point out that, when the volatility parameter $\sigma$ and/or the participation level $\eta$ are low, the price $c$ of the call option defined by relation (14) of Section 3 practically vanishes and then the rounded values of $i$ and $\exp \{r\}-1$ 
coincide, in terms of basis points. Moreover, observe that with a market rate of $3 \%$ and a volatility coefficient of $15-20 \%$, there are non negative solutions for $\mathrm{i}$ only when $\eta \leq 30 \%$ (see Table 1), whilst, for instance, when $\mathrm{r}=10 \%$ and $\sigma=15 \%$, a participation level between $70 \%$ and $80 \%$ leads to a fair technical rate between $4.28 \%$ and $0.7 \%$ (see Table 2 ).

\subsection{Solutions with respect to the participation level $\eta$}

Assume now that, given $r>0$, the insurance company has already fixed a technical interest rate $i \in[0, \exp \{r\}-1)$, and chosen a reference portfolio with volatility coefficient $\sigma>0$. We are then concerned with the determination of a participation level $\eta$, between 0 and 1 , such that the contract is fair.

As in the case analysed in the previous subsection, we observe first of all that

$$
\frac{\partial g}{\partial \eta}=c(r, i, \eta, \sigma)+(i / \eta) \exp \{-r\} F\left(d_{2}\right)>0,
$$

i.e., that $\mathrm{g}$ is strictly increasing also with respect to $\eta$. Moreover:

$$
\begin{gathered}
\inf _{\eta>0} g(r, i, \eta, \sigma)=\lim _{\eta \rightarrow 0} g(r, i, \eta, \sigma)=\exp \{-r\}(1+i)-1<0, \\
\sup _{\eta<1} g(r, i, \eta, \sigma)=\lim _{\eta \rightarrow 1} g(r, i, \eta, \sigma)=\exp \{-r\}(1+i)+c(r, i, 1, \sigma)-1>0 .
\end{gathered}
$$

The first inequality follows immediately from the fact that $i<\exp \{r\}-1$. To establish the second one define

$$
\begin{aligned}
& z(r, i, \sigma):=\exp \{-r\}(1+i)+c(r, i, 1, \sigma)-1, \\
& f(y):=F^{\prime}(y)=\frac{1}{\sqrt{2 \pi}} \exp \left\{-y^{2} / 2\right\}
\end{aligned}
$$

and observe that

$$
\frac{\partial z}{\partial \sigma}=\exp \{-r\}(1+i) f([r-\ln (1+i)] / \sigma-\sigma / 2)>0,
$$

i.e., $\mathrm{z}$ is strictly increasing with respect to $\sigma$, and

$$
\inf _{\sigma>0} z(r, i, \sigma)=\lim _{\sigma \rightarrow 0} z(r, i, \sigma)=0 .
$$

Therefore, given $r>0, \sigma>0$, and $i \in[0, \exp \{r\}-1)$, there is a unique $\eta \in(0,1)$ such that $g(r, i, \eta, \sigma)=0$.

Tables 3 and 4 report some examples of solutions to the fairness condition with respect to $\eta$ for given values of $i$ and $\sigma$ when $r$ is equal to $3 \%$ and to $10 \%$ respectively. 
TABLE 3

SOLUTIONS WITH RESPECT TO THE PARTICIPATION LEVEL $\eta$ (B.P.) WHEN $r=0.03$

\begin{tabular}{ccccccccc}
\hline \hline $\begin{array}{c}\boldsymbol{\sigma} \\
\mathbf{i}\end{array}$ & $\mathbf{0 . 0 5}$ & $\mathbf{0 . 1 0}$ & $\mathbf{0 . 1 5}$ & $\mathbf{0 . 2 0}$ & $\mathbf{0 . 2 5}$ & $\mathbf{0 . 3 0}$ & $\mathbf{0 . 3 5}$ & $\mathbf{0 . 4 0}$ \\
\hline $\mathbf{0 . 0 0 0}$ & 7806 & 5295 & 3948 & 3140 & 2604 & 2225 & 1943 & 1724 \\
$\mathbf{0 . 0 0 5}$ & 7414 & 4929 & 3645 & 2883 & 2383 & 2029 & 1767 & 1564 \\
$\mathbf{0 . 0 1 0}$ & 6951 & 4522 & 3313 & 2606 & 2144 & 1819 & 1579 & 1394 \\
$\mathbf{0 . 0 1 5}$ & 6394 & 4061 & 2944 & 2299 & 1882 & 1589 & 1374 & 1208 \\
$\mathbf{0 . 0 2 0}$ & 5696 & 3516 & 2516 & 1948 & 1583 & 1330 & 1143 & 1000 \\
$\mathbf{0 . 0 2 5}$ & 4741 & 2818 & 1980 & 1514 & 1218 & 1013 & 864 & 750 \\
$\mathbf{0 . 0 3 0}$ & 2744 & 1494 & 1000 & 737 & 574 & 463 & 384 & 324 \\
\hline \hline
\end{tabular}

TABLE 4

SOLUTIONS WITH RESPECT TO THE PARTICIPATION LEVEL $\eta$ (B.P.) WHEN $r=0.1$

\begin{tabular}{ccccccccc}
\hline \hline $\boldsymbol{\sigma}$ & $\mathbf{0 . 0 5}$ & $\mathbf{0 . 1 0}$ & $\mathbf{0 . 1 5}$ & $\mathbf{0 . 2 0}$ & $\mathbf{0 . 2 5}$ & $\mathbf{0 . 3 0}$ & $\mathbf{0 . 3 5}$ & $\mathbf{0 . 4 0}$ \\
$\mathbf{i}$ & & & & & & & & \\
\hline $\mathbf{0 . 0 0 0}$ & 9958 & 9232 & 8155 & 7171 & 6354 & 5687 & 5138 & 4684 \\
$\mathbf{0 . 0 0 5}$ & 9945 & 9154 & 8045 & 7052 & 6234 & 5570 & 5026 & 4575 \\
$\mathbf{0 . 0 1 0}$ & 9929 & 9069 & 7929 & 6927 & 6110 & 5449 & 4910 & 4465 \\
$\mathbf{0 . 0 1 5}$ & 9909 & 8977 & 7807 & 6798 & 5982 & 5325 & 4791 & 4352 \\
$\mathbf{0 . 0 2 0}$ & 9884 & 8878 & 7679 & 6664 & 5849 & 5198 & 4670 & 4235 \\
$\mathbf{0 . 0 2 5}$ & 9854 & 8771 & 7544 & 6523 & 5712 & 5066 & 4544 & 4116 \\
$\mathbf{0 . 0 3 0}$ & 9817 & 8655 & 7402 & 6377 & 5570 & 4930 & 4415 & 3993 \\
$\mathbf{0 . 0 3 5}$ & 9772 & 8529 & 7251 & 6224 & 5422 & 4789 & 4281 & 3867 \\
$\mathbf{0 . 0 4 0}$ & 9718 & 8392 & 7092 & 6064 & 5268 & 4643 & 4143 & 3737 \\
$\mathbf{0 . 0 4 5}$ & 9654 & 8244 & 6923 & 5896 & 5107 & 4491 & 4001 & 3602 \\
$\mathbf{0 . 0 5 0}$ & 9577 & 8082 & 6743 & 5718 & 4938 & 4333 & 3852 & 3462 \\
$\mathbf{0 . 0 5 5}$ & 9485 & 7906 & 6551 & 5531 & 4762 & 4167 & 3697 & 3317 \\
$\mathbf{0 . 0 6 0}$ & 9376 & 7712 & 6345 & 5333 & 4575 & 3994 & 3535 & 3165 \\
$\mathbf{0 . 0 6 5}$ & 9246 & 7499 & 6123 & 5121 & 4378 & 3810 & 3364 & 3006 \\
$\mathbf{0 . 0 7 0}$ & 9091 & 7262 & 5882 & 4894 & 4167 & 3615 & 3184 & 2838 \\
$\mathbf{0 . 0 7 5}$ & 8904 & 6998 & 5618 & 4647 & 3941 & 3407 & 2991 & 2659 \\
$\mathbf{0 . 0 8 0}$ & 8677 & 6698 & 5325 & 4377 & 3694 & 3181 & 2783 & 2467 \\
$\mathbf{0 . 0 8 5}$ & 8396 & 6351 & 4995 & 4076 & 3421 & 2932 & 2556 & 2257 \\
$\mathbf{0 . 0 9 0}$ & 8038 & 5938 & 4611 & 3731 & 3110 & 2651 & 2299 & 2022 \\
$\mathbf{0 . 0 9 5}$ & 7556 & 5422 & 4144 & 3316 & 2741 & 2319 & 1999 & 1747 \\
$\mathbf{0 . 1 0 0}$ & 6825 & 4699 & 3510 & 2763 & 2254 & 1887 & 1609 & 1393 \\
$\mathbf{0 . 1 0 5}$ & 4608 & 2790 & 1932 & 1437 & 1116 & 894 & 731 & 607 \\
\hline \hline
\end{tabular}


As far as the results reported in Tables 3 and 4 are concerned, we observe that, when $r=3 \%$, a reference portfolio with a medium/high volatility produces a very low fair participation level. For instance, if $\sigma=30 \%$, a technical rate between 0 and 3\% gives rise to a fair participation level between $22.25 \%$ and $4.63 \%$ (see Table 3). When instead $\mathrm{r}=10 \%$, the fair participation levels are obviously higher. For instance, a 3\%-value of the technical rate, very common in Italy at the end of the seventies, when the policies with profits were introduced, leads to fair participation levels between $98.17 \%$ and $39.93 \%$, corresponding to volatility coefficients between $5 \%$ and $40 \%$ (see Table 4).

\subsection{Solutions with respect to the volatility coefficient $\sigma$}

We analyse now the problem of finding a volatility coefficient $\sigma>0$ in order to satisfy the fairness relation, given a market rate $r>0$ and once the insurance company has fixed a participation level $\eta \in(0,1)$ and a technical rate $i \in[0, \exp \{r\}-1)$.

Once again, we exploit the strict monotonicity of $g$ with respect to the third control-parameter $\sigma$. Observe, in fact, that

$$
\frac{\partial g}{\partial \sigma}=\eta(1+i / \eta) \exp \{-r\} f\left(d_{2}\right)>0
$$

Moreover,

$$
\inf _{\sigma>0} g(r, i, \eta, \sigma)=\lim _{\sigma \rightarrow 0} g(r, i, \eta, \sigma)=\left\{\begin{array}{c}
{[1-\exp \{-r\}](\eta-1)<0} \\
\text { if } i / \eta<\exp \{-r\}-1 \\
\exp \{-r\}(1+i)-1<0 \\
\text { if } i / \eta \geq \exp \{-r\}-1
\end{array}\right.
$$

and

$$
\sup _{\sigma>0} g(r, i, \eta, \sigma)=\lim _{\sigma \rightarrow+\infty} g(r, i, \eta, \sigma)=\exp \{-r\}(1+i)+\eta-1
$$

Then a necessary and sufficient condition for the existence of a unique solution in $\sigma$ to the equation $g(r, i, \eta, \sigma)=0$ is $\exp \{-r\}(1+i)+\eta-1>0$. This condition produces the following (strictly positive) lower bound for $\eta$ :

$$
\eta>1-\exp \{-r\}(1+i)
$$

Summing up, given $r>0, i \in[0, \exp \{r\}-1)$ and $\eta \in(1-\exp \{-r\}(1+i), 1)$, there exists a unique $\sigma>0$ such that the contract is fair.

Some numerical solutions with respect to $\sigma$ for given values of $i$ and $\eta$ are reported in Tables 5 and 6 , where $\mathrm{r}$ is $3 \%$ and $10 \%$ respectively. 
TABLE 5

SOLUTIONS WITH RESPECT TO THE VOLATILITY COEFFICIENT $\sigma$ (B.P.) WHEN $r=0.03$

\begin{tabular}{ccrrrrrrrr}
\hline $\begin{array}{c}\boldsymbol{\eta} \\
\mathbf{i}\end{array}$ & $\mathbf{0 . 1}$ & $\mathbf{0 . 2}$ & $\mathbf{0 . 3}$ & $\mathbf{0 . 4}$ & $\mathbf{0 . 5}$ & $\mathbf{0 . 6}$ & $\mathbf{0 . 7}$ & $\mathbf{0 . 8}$ & $\mathbf{0 . 9}$ \\
\hline $\mathbf{0 . 0 0 0}$ & 7296 & 3387 & 2113 & 1475 & 1087 & 823 & 628 & 472 & 333 \\
$\mathbf{0 . 0 0 5}$ & 6478 & 3049 & 1907 & 1331 & 979 & 740 & 562 & 420 & 293 \\
$\mathbf{0 . 0 1 0}$ & 5670 & 2702 & 1693 & 1180 & 867 & 652 & 493 & 366 & 253 \\
$\mathbf{0 . 0 1 5}$ & 4855 & 2338 & 1466 & 1020 & 747 & 559 & 420 & 309 & 210 \\
$\mathbf{0 . 0 2 0}$ & 4002 & 1943 & 1218 & 845 & 615 & 457 & 340 & 247 & 164 \\
$\mathbf{0 . 0 2 5}$ & 3039 & 1484 & 927 & 639 & 461 & 338 & 248 & 175 & 112 \\
$\mathbf{0 . 0 3 0}$ & 1501 & 727 & 446 & 300 & 210 & 149 & 103 & 67 & 37 \\
\hline \hline
\end{tabular}

TABLE 6

SOLUTIONS WITH RESPECT TO THE VOLATILITY COEFFICIENT $\sigma$ (B.P.) WHEN $r=0.1$

\begin{tabular}{cccccccccc}
\hline \hline $\boldsymbol{\eta}$ & $\mathbf{0 . 1}$ & $\mathbf{0 . 2}$ & $\mathbf{0 . 3}$ & $\mathbf{0 . 4}$ & $\mathbf{0 . 5}$ & $\mathbf{0 . 6}$ & $\mathbf{0 . 7}$ & $\mathbf{0 . 8}$ & $\mathbf{0 . 9}$ \\
$\mathbf{i}$ & & & & & & & & & \\
\hline $\mathbf{0 . 0 0 0}$ & 39052 & 11902 & 7183 & 4962 & 3643 & 2753 & 2097 & 1574 & 1110 \\
$\mathbf{0 . 0 0 5}$ & 33286 & 11413 & 6933 & 4800 & 3526 & 2665 & 2029 & 1521 & 1070 \\
$\mathbf{0 . 0 1 0}$ & 29577 & 10939 & 6685 & 4638 & 3410 & 2576 & 1960 & 1468 & 1030 \\
$\mathbf{0 . 0 1 5}$ & 26785 & 10477 & 6440 & 4476 & 3293 & 2488 & 1891 & 1414 & 990 \\
$\mathbf{0 . 0 2 0}$ & 24522 & 10027 & 6197 & 4315 & 3175 & 2399 & 1822 & 1361 & 950 \\
$\mathbf{0 . 0 2 5}$ & 22603 & 9587 & 5955 & 4153 & 3058 & 2309 & 1753 & 1306 & 910 \\
$\mathbf{0 . 0 3 0}$ & 20928 & 9156 & 5714 & 3991 & 2939 & 2219 & 1682 & 1252 & 869 \\
$\mathbf{0 . 0 3 5}$ & 19433 & 8732 & 5474 & 3829 & 2820 & 2128 & 1611 & 1197 & 828 \\
$\mathbf{0 . 0 4 0}$ & 18076 & 8314 & 5234 & 3665 & 2700 & 2036 & 1540 & 1141 & 786 \\
$\mathbf{0 . 0 4 5}$ & 16827 & 7901 & 4993 & 3501 & 2579 & 1943 & 1467 & 1085 & 744 \\
$\mathbf{0 . 0 5 0}$ & 15663 & 7491 & 4751 & 3334 & 2455 & 1848 & 1394 & 1028 & 702 \\
$\mathbf{0 . 0 5 5}$ & 14568 & 7083 & 4507 & 3165 & 2330 & 1752 & 1319 & 969 & 659 \\
$\mathbf{0 . 0 6 0}$ & 13526 & 6675 & 4260 & 2994 & 2203 & 1654 & 1242 & 910 & 615 \\
$\mathbf{0 . 0 6 5}$ & 12525 & 6264 & 4009 & 2818 & 2072 & 1553 & 1164 & 849 & 570 \\
$\mathbf{0 . 0 7 0}$ & 11554 & 5849 & 3753 & 2638 & 1938 & 1450 & 1083 & 787 & 524 \\
$\mathbf{0 . 0 7 5}$ & 10601 & 5426 & 3488 & 2452 & 1799 & 1343 & 999 & 722 & 477 \\
$\mathbf{0 . 0 8 0}$ & 9652 & 4990 & 3213 & 2257 & 1653 & 1230 & 912 & 655 & 428 \\
$\mathbf{0 . 0 8 5}$ & 8692 & 4533 & 2922 & 2050 & 1498 & 1111 & 819 & 584 & 376 \\
$\mathbf{0 . 0 9 0}$ & 7694 & 4044 & 2608 & 1826 & 1330 & 981 & 719 & 507 & 321 \\
$\mathbf{0 . 0 9 5}$ & 6613 & 3497 & 2254 & 1574 & 1140 & 836 & 607 & 422 & 261 \\
$\mathbf{0 . 1 0 0}$ & 5327 & 2829 & 1818 & 1262 & 907 & 658 & 470 & 320 & 190 \\
$\mathbf{0 . 1 0 5}$ & 2740 & 1448 & 916 & 622 & 435 & 304 & 207 & 130 & 66 \\
\hline \hline $\mathbf{0}$ & & & & & & & & & \\
\hline
\end{tabular}


Once again, we choose the extreme scenarios considered in Tables 5 and 6 in order to catch some numerical feelings about our findings. When $r=3 \%$ (a scenario not far from the Italian one at the present time) and the participation level is rather high, then a fair pricing is attainable only with the choice of a reference portfolio characterized by a very low volatility. For instance, if $\eta$ is between $70 \%$ and $90 \%$, a fair pricing would require a volatility coefficient between $6.28 \%$ and $3.33 \%$ for $\mathrm{i}=0$, and respectively between $1.03 \%$ and $0.37 \%$ for $\mathrm{i}=3 \%$ (see Table 5). When instead $\mathrm{r}=10 \%$, a technical rate of $3 \%$ and a participation level between $70 \%$ and $90 \%$ lead to a fair volatility coefficient between $16.82 \%$ and $8.69 \%$ (see Table 6 ).

\section{HedGiNG STRATEGiES}

In the previous sections we have focussed strongly on a price, that is the fair premium for an Italian-style life insurance participating policy, and on the conditions under which this price coincides with that actually requested by Italian insurance companies. However, the fair price is only relevant if some hedging strategy is in force. Then, in this section, we hint at possible hedging strategies and at the practical problems connected with their fulfilment.

To this end, we first observe that, at the beginning of the $t$-th year of contract (i.e., at time $t-1$ for $t=1, \ldots, T$ ), if the insured is alive, the amount invested in the reference portfolio for the policy under consideration is required (by regulatory rules) to equal at least the mathematical reserve, which we denote by $V_{t-1}$, plus the $t$-th premium $P_{t-1}$ in the case of periodical premium contracts. If the insured dies during the year, the company must pay (at time $t$ ) the benefit $C_{t}$; otherwise it has to set aside the mathematical reserve $V_{t}$, given recursively by the following relations:

$$
\left\{\begin{array}{l}
V_{0}=U \\
V_{t-1}(1+i)\left(1+\delta_{t}\right)=q_{x+t-1} C_{t}+p_{x+t-1} V_{t} \quad t=1,2, \ldots, T
\end{array}\right.
$$

in the case of single premium contracts, and

$$
\left\{\begin{array}{l}
\mathrm{V}_{0}=0 \\
\left(\mathrm{~V}_{\mathrm{t}-1}+\mathrm{P}_{\mathrm{t}-1}\right)(1+\mathrm{i})\left(1+\delta_{\mathrm{t}}\right)=\mathrm{q}_{\mathrm{x}+\mathrm{t}-1} \mathrm{C}_{\mathrm{t}}+\mathrm{p}_{\mathrm{x}+\mathrm{t}-1} \mathrm{~V}_{\mathrm{t}} \quad \mathrm{t}=1,2, \ldots, \mathrm{T}
\end{array}\right.
$$

in the case of periodical premiums, where $\mathrm{U}, \mathrm{C}_{t}, \mathrm{P}_{t}, \delta_{t}$ are given by expressions (1) to (7) of Section 2, $\mathrm{p}_{\mathrm{x}+\mathrm{t}-1}$ is the probability for a life aged $\mathrm{x}+\mathrm{t}-1$ to be alive at age $x+t$, and $q_{x+t-1}=1-p_{x+t-1}$.

Moreover, if $t<T$, the mathematical reserve $V_{t}$ is strictly less than $C_{t}$, so that, at the beginning of each year of contract (the last one excepted), the company has to face both a financial and a mortality risk concerning the 
coming year. Taking into account that the mortality risk can be (substantially) hedged by diversification arguments, we focus now on the financial risk, that arises since the company gets a random return at rate $\mathrm{g}_{\mathrm{t}}$ on its investments, while it promises the policyholder a return at rate $(1+\mathrm{i})\left(1+\delta_{t}\right)-1$ $=\max \left\{i, \eta g_{t}\right\}$. Then, in "good years", the company realizes a "gain" at rate

$$
\begin{cases}g_{t}-i & \text { if } i \leq g_{t} \leq i / \eta \\ g_{t}(1-\eta) & \text { if } g_{t}>i / \eta\end{cases}
$$

on the (average) amount $\mathrm{V}_{t-1}$ (plus $\mathrm{P}_{\mathrm{t}-1}$ in the case of periodical premiums), while it suffers a "loss" at rate $\mathrm{i}-\mathrm{g}_{\mathrm{t}}$ on the same amount in "bad years".

To hedge this risk, at least from a theoretical point of view, the company could follow a dynamic strategy that guarantees, for instance, the amounts $R_{t-1} \max \left\{i-g_{t}, 0\right\}$ at the end of each year of contract, where $R_{t-1}=V_{t-1}$ for single premium contracts and $R_{t-1}=V_{t-1}+P_{t-1}$ for periodic premium ones. This strategy would require to "buy", at each time $t-1(t=1,2, \ldots, T)$, contingentclaims with time to maturity 1 and final payoff $R_{t-1} \max \left\{i-g_{t}, 0\right\}$. Recalling the definition of $g_{t}$, one has

$$
\mathrm{R}_{\mathrm{t}-1} \max \left\{\mathrm{i}-\mathrm{g}_{\mathrm{t}}, 0\right\}=\mathrm{R}_{\mathrm{t}-1} \mathrm{G}_{\mathrm{t}-1} \max \left\{\mathrm{G}_{\mathrm{t}-1}(1+\mathrm{i})-\mathrm{G}_{\mathrm{t}}, 0\right\},
$$

which corresponds to the payoff of $R_{t-1} G_{t-1}$ European put options on 1 unit of the reference fund with maturity $t$ and exercise price $G_{t-1}(1+i)$.

However, from a practical point of view this strategy implies at least two serious problems. First of all, the reference portfolio is usually an internal one, and therefore no traded options with this particular underlying asset are available. Anyway, if this portfolio mimics a benchmark, there could be traded options on the benchmark, and the company could use the gains realized in good years for buying this kind of options. The second problem arises from the fact that there could be a sequence of bad years, in which these options are deeply in the money, with a consequent prohibitive cost for the company. Moreover, in this way, the financial risk is simply shifted from the end to the beginning of each year, when the options are bought.

Alternatively, the company could try to replicate the options by means of dynamic strategies involving frequent rebalancing, according to the market conditions, of the proportion between risky assets and default-free bonds in the portfolio. For a detailed analysis of such strategies see, for instance, Lindset (2000). Obviously also these strategies are not costless, either because there are transaction costs in the "real world", or because a too conservative investment policy aimed at eliminating the financial risk associated to the minimum guarantee provision could produce, in good years, a worse performance than the policy of a more "aggressive" competitor, and this fact would compromise the marketability of the products offered by the company under consideration. 


\section{Concluding Remarks}

In this paper we have analysed a life insurance endowment policy, paid either by a single premium at issuance or by a sequence of periodical premiums, in which both the benefit and the periodical premiums are annually adjusted according to the performance of a special investment portfolio. The premium calculation technique and the adjustment mechanism are defined in such a way that a minimum interest rate is guaranteed to the policyholder and, moreover, a special bonus is annually credited to the mathematical reserve of the policy. These features introduce in the contract some embedded options, of European style, that can be priced in a contingent-claims framework once an independence assumption allows us to keep apart the financial risk from the mortality one. Under the Black and Scholes model for the evolution of the reference portfolio and exploiting the martingale approach, we derive a very simple closed-form relation that characterizes "fair" contracts, i.e., contracts priced consistently with the usual assumptions on financial markets and, in particular, with no-arbitrage. This relation links together the contractual parameters (i.e., the minimum interest rate guaranteed and a "participation" coefficient) with the market interest rate and the riskiness of the reference portfolio.

Undoubtedly our valuation model is very simple, although it includes almost all the features of Italian participating policies. However, taking into account that life insurance policies are usually long-term contracts and bearing in mind the experience on the evolution of the market interest rates in the last two decades, it must be admitted that a framework with deterministic interest rates, such as the Black and Scholes one, is not suitable to represent the real world. Therefore a natural extension of the present paper is certainly the inclusion of stochastic interest rates, as like as stochastic volatility. Notwithstanding this, our model can be useful to an insurance company for fixing the participation level, once all the remaining parameters are given. This model is actually based on the assumption of a constant participation level, although the insurers usually reserve themselves the right to fix year by year the value of this parameter. Therefore, if there is a change in the market rate (or in the volatility of the reference fund), the model can be applied with the new parameters in order to update the participation level, provided that the market rate does not fall below the technical one.

Another issue connected to participating policies is the presence of a surrender option. Since the surrender values of Italian products used to include some penalties, justified by several reasons (adverse selection, loss of future earnings, ...), this option hardly ever turned out to be in the money. Nontheless many policyholders, for personal reasons, were forced to surrender their contracts, so that such penalties produced a certain degree of dissatisfaction. Now that the competition among insurance companies and banks has become particularly aggressive, it is likely to expect that the surrender conditions play a crucial rôle in this competition. Then an accurate assessment of the surrender values and, consequently, the fair valuation of the American-style surrender option, constitute important topics to be addressed in the near future. 


\section{ACKNOWLEDGEMENTS}

The author thanks two anonymous referees for helpful comments and suggestions; of course, the usual disclaimer applies. Financial support from Regione Autonoma Friuli-Venezia Giulia on "Modelli Matematici Innovativi per lo Studio dei Rischi Finanziari e Assicurativi" is gratefully acknowledged.

\section{REFERENCES}

Bacinello, A.R. (2000). Fair Pricing of Life Insurance Participating Policies with a Minimum Interest Rate Guaranteed. Proceedings of the Tenth AFIR International Colloquium, Tromsø, $1-28$.

Black, F. and Scholes, M. (1973). The Pricing of Options and Corporate Liabilities. Journal of Political Economy 81, 637-654.

BlazenKo, G.W., Boyle, P.P. and Newport, K.E. (1990). Valuation of Tandem Options. Advances in Futures and Options Research, vol. 4, JAI Press Inc., 39-49.

BoYLE, P.P. and SCHWARTZ, E.S. (1977). Equilibrium Prices of Guarantees under Equity-Linked Contracts. Journal of Risk and Insurance 44, 639-660.

Brennan, M.J. and Schwartz, E.S. (1976). The Pricing of Equity-Linked Life Insurance Policies with an Asset Value Guarantee. Journal of Financial Economics 3, 195-213.

BrENNAN, M.J. and SCHWARTZ, E.S. (1979a). Alternative Investment Strategies for the Issuers of Equity Linked Life Insurance Policies with an Asset Value Guarantee. Journal of Business 52, 63-93.

Brennan, M.J. and Schwartz, E.S. (1979b). Pricing and Investment Strategies for EquityLinked Life Insurance. The S.S. Huebner Foundation for Insurance Education, Wharton School, University of Pennsylvania, Philadelphia.

BRIYS, E. and DE VARENNE, F. (1997). On the Risk of Life Insurance Liabilities: Debunking Some Common Pitfalls. Journal of Risk and Insurance 64, 673-694.

Grosen, A. and JørGensen, P.L. (2000). Fair Valuation of Life Insurance Liabilities: The Impact of Interest Rate Guarantees, Surrender Options, and Bonus Policies. Insurance: Mathematics and Economics 26, 37-57.

Hansen, M. and Miltersen, K.R. (2000). Minimum Rate of Return Guarantees: The Danish Case. Proceedings of the Tenth AFIR International Colloquium, Tromsø, 309-329.

HARRISON, M.J. and KREPS, D. (1979). Martingales and Arbitrage in Multiperiod Securities Markets. Journal of Economic Theory 20, 381-408.

HARrison, M.J. and PliskA, S. (1981). Martingales and Stochastic Integrals in the Theory of Continuous Trading. Stochastic Processes and Their Applications 11, 215-260.

Harrison, M.J. and Pliska, S. (1983). A Stochastic Calculus Model of Continuous Trading: Complete Markets. Stochastic Processes and Their Applications 15, 313-316.

Jensen, B., Jørgensen, P.L., and Grosen, A. (2000). A Finite Difference Approach to the Valuation of Path Dependent Life Insurance Liabilities. Proceedings of the Tenth AFIR International Colloquium, Tromsø, 279-307.

LINDSET, S. (2000). Hedging Strategies for Rate of Return Guarantees on Multi-period Assets. Proceedings of the Tenth AFIR International Colloquium, Tromsø, 447-467.

Miltersen, K.R. and Persson, S.-A. (2000a). Guaranteed Investment Contracts: Distributed and Undistributed Excess Returns. Working Paper 2000/1, Department of Finance and Management Science, Norwegian School of Economics and Business Administration.

Miltersen, K.R. and Persson, S.-A. (2000b). A Note on Interest Rate Guarantees and Bonus: The Norwegian Case. Proceedings of the Tenth AFIR International Colloquium, Tromse, 507516.

Pentikäinen, T. (1968). Linking Life and Private Pension Insurance to Price Index. Transactions of the 18th International Congress of Actuaries, München, vol. 2, 847-859. 
VASICEK, O. (1977). An Equilibrium Characterization of the Term Structure. Journal of Financial Economics 5, 177-188.

WiLkiE, A.D. (1987). An Option Pricing Approach to Bonus Policy. Journal of the Institute of Actuaries 114, 21-77.

\section{AnNA Rita BACINELlo}

Dipartimento di Matematica Applicata alle Scienze Economiche, Statistiche ed Attuariali "Bruno de Finetti", Università degli Studi di Trieste

Piazzale Europa, 1

I-34127 Trieste, Italia

E-mail:bacinel@univ.trieste.it 